\title{
Investigate C+L Band EDFA/Raman Amplifiers by Using the Same Pump Lasers
}

\author{
Shien-Kuei Liaw ${ }^{1}$, Keang-Po Ho ${ }^{2}$. Cheng-Kai Huang ${ }^{1}$, Wen-Ting Chen ${ }^{1}$, \\ Yuan-Lung Hsiao ${ }^{1}$ and Ing-Ge Lai ${ }^{1}$ \\ ${ }^{1}$ Dept. of Electronic Engineering, National Taiwan University of Sci. and Tech., \\ Taipei 106, Taiwan,skliaw@et.ntust.edu.tw \\ ${ }^{2}$ Inst.of Communication Engineering and Dept. of Electrical Engineering, \\ National Taiwan University, Taipei 106, Taiwan, kpho@ieee.org
}

\begin{abstract}
A hybrid $\mathrm{C}+\mathrm{L}$ band erbium-doped fiber amplifier/Raman fiber amplifier (EDFA/RFA) is constructed by sharing common $1480 \mathrm{~nm}$ pump source(s). It is based on three-level amplification mechanism in the $\mathrm{C}$ band and Raman shift amplification mechanism the in L band, respectively. The gain spectra of $\mathrm{C}+\mathrm{L}$ band are flattened by optimally dividing the pump power ratio of 1:29 for EDFA/RFA. Gain variation is $14 \pm 1 \mathrm{~dB}$ for the C band EDFA and $13 \pm 1.5 \mathrm{~dB}$ for the L band RFA without gain equalization. The low noise, hybrid $\mathrm{C}+\mathrm{L}$ band EDFA/RFA may find vast applications in WDM system and lightwave transmission.
\end{abstract}

Keywords: Raman fiber amplifier, hybrid amplifier, pump share, noise figure, lightwave communication.

\section{Introduction}

Optical amplifiers are indispensable optical components to compensate for fibre loss in long-haul transmission system [1-2]. With a wide gain bandwidth, typical Erbium-doped fibre amplifiers (EDFAs) enable wavelength-division-multiplexed (WDM) based optical networking by amplifying all channels simultaneously without signal distortion and crosstalk. EDFA is suitable to operate at the conventional (C) band from about 1530 to $1565 \mathrm{~nm}$ [1]. Since the entire $C$ band of EDFA is fully utilized, the need for more optical channels and wider optical bandwidth urges EDFA technology to develop beyond its present limits. To extend the optical bandwidth and increase the number of WDM channels, L-band optical amplifiers are used to operate in longer wavelength from about 1570 to $1605 \mathrm{~nm}$. EDFA by itself has a very low-gain at the L-band, most realizations of L-band EDFA implement a long length of erbium-doped fibre (EDF) to pump up its gain. A typical L-band EDFA also has larger noise figure than C-band EDFA [3]. Unlike EDFA have the problems of umpumped amplifier attenuations and the operation wavelength constrained at 1.53-1.56 $\mu \mathrm{m}$ region, Raman fiber amplifier (RFA) has merit of arbitrary gain bandwidth, which were recently being recognized as an enabling technology for high capacity and long-haul density wavelength-division- multiplexing (DWDM) systems. RFA can be used to amplify not only the C-band, but also the S-, L- and other bands, depending on the usage of the pumped wavelengths [4]. RFA has several advantages including lower noise figure (NF), flexibility on the selection of gain medium, and wide gain bandwidth [5], especially that RFA has the capability to "distribute" the gain over a long distance in the transmission fiber. The difference between maximum and minimum intensity in a span can be reduced without reducing the span length, avoiding problems of noise and nonlinearity. Thus, L-band optical amplifier is better to adopt RFA rather than L band EDFA [6-7]. Recently, we have demonstrated a dual-pump RFA with $24 \mathrm{~dB}$ effective gain using dual pump scheme [8]. But the gain spectrum is restricted in the $\mathrm{C}$ band. In this paper, a novel and cost effective design fiber amplifier is proposed to extend the gain spectra by using the same pump lasers for the hybrid C/L band EDFA/RFA.

\section{Principle of the proposed hybrid $\mathbf{C}+\mathbf{L}$ band amplifier}

Stimulated Raman scattering is a loss mechanism when it steals energy away from the shorter wavelength and transfers it to longer wavelength. It means that Raman amplification can extract energy from a pump beam to amplify a weak optical signal at another wavelength [9]. The Raman effect is an interaction between light and a material that causes some of the light to be shifted to a different wavelength, usually to a longer wavekength. The amount of shift depends on the material and the energy of an excitation like an automatic vibration. If the Raman pump is at $1480 \mathrm{~nm}$, the corresponding gain peaks can be calculated based on the following equation:

$$
\lambda=-\lambda \quad \mathrm{f} / \mathrm{f}=-\lambda^{2} \quad \mathrm{f} / \mathrm{C}=94.9 \mathrm{~nm}
$$

where $\mathrm{f}=-13 \mathrm{THz} . \quad \lambda=94.9 \mathrm{~nm}$ means that the pump wavelength is about $94.9 \mathrm{~nm}$ shorter than the maximum amplified wavelngth at $1574.9 \mathrm{~nm}$. Fig. 1 . shows the concept of using only one pumping laser for simultaneous C-EDFA and L-RFA amplifications. 
With only a pump wavelength of $1480 \mathrm{~nm}$, the C-band EDFA is based on three-level amplification mechanism in erbium ions, while the L-band RFA is based on Raman shift amplification mechanism. Fig. 2 shows schematic diagram of the proposed hybrid $\mathrm{C}+\mathrm{L}$ band EDFA/RFA shared the same pump. At the input of the optical amplifier, a C/L-band WDM coupler is used to separate the WDM signals to $\mathrm{C}$ and $\mathrm{L}$ bands. The C-band signals are amplified by an EDFA and the L-band signals are amplified by a RFA. They share the same pump source @ 1480 nm, which is splitted by a variable-ratio coupler to equalize the gain of $\mathrm{C}$ - and L- bands. To increase the gain of EDFA and reduce the polarization dependent gain (PDG) of RFA, the backward pump configuration is adopted by inject the pump sources from the opposite ends of the gain fibers for the hybrid $\mathrm{C}+\mathrm{L}$ band amplifier. Since EDFA has a larger gain efficiency than RFA, a smaller ratio of pump power is used to provide gain for the C-band EDFA. The splitting ratio of the pump laser between the C-band EDFA and L-band RFA is optimised based on their gain characteristics such as physical properties of EDF and dispersion compensation fiber (DCF), as well as the pumping efficiency. The gain of $\mathrm{C}$ band EDFA is given by:

$$
g=\frac{\sigma_{e m} \tau}{h v_{P}} \frac{P_{a b s}}{A_{e f f}} \frac{F}{\eta_{P}}
$$

where $\sigma_{e m}$ is the emission cross-section, $\tau$ is the upper-state lifetime, $h v_{p}$ is the pump photon energy, $A_{\text {eff }}$ is the fibre core area, $P_{a b s}$ is the absorbed pump power, $F$ is the overlap integral between the pump and signal fields in the transverse dimensions, and $\eta_{P}$ is the fractional pump energy. The gain of the RFA ( $\mathrm{L}$ band) is defined as [10].

$$
G_{A}=\exp \left(\frac{g_{R} P_{0} L_{e f f}^{p}}{A_{e f f}}\right)
$$

where $g_{R}$ is the Raman gain coefficient as a function of the wavelength difference between signal and pump, $P_{0}$ is the pump power at the amplifier input, and $L_{e f f}^{p}$ is the pump effective length. The $\mathrm{dB}$ form of the RFA gain can be expressed as:

$$
G_{A}^{d B}=10 \log \left[\exp \left(\frac{g_{R} L_{e f f}}{A_{\text {eff }}} \times P_{0}\right)\right]=10 \times \frac{g_{R} L_{e f f}}{A_{\text {eff }}} \times P_{0} \log e
$$

The values of $g$ for the $C$ band in equ. (2) and $G_{A}$ for the $L$ band in equ. (3) are set equal. Noise figure (NF) of EDFA is defined as [11]:

$$
N F(\lambda)=\frac{1}{G}\left[1+\frac{\lambda^{3} P_{A S E}(\lambda)}{h c^{2} \Delta \lambda}\right]
$$

where $G$ is the EDFA gain, $h$ is the Planck's constant, and $c$ is the speed of light in vacuum. To express the RFA noise using the equivalent noise figure ( $\mathrm{NFeq}$ ), which is derived from the NF cascading formula known as the Friis formulation [12]

$$
N F_{e q}=\frac{1}{G_{R}}+\frac{2 P_{A S E}}{G_{R} h v B_{0}}
$$

where $\boldsymbol{h} \boldsymbol{v}$ is the photon energy and Bo is optical measurement bandwidth. NFeq represents the NF that would experience a discrete pre-amplifier of gain $G_{R}$ and generating the same noise than the considered distributed RFA [13].

\section{Experimental setup}

The experimental setup to demonstrate the proposed hybrid $\mathrm{C}+\mathrm{L}$ band EDFA/RFA is shown in Fig. 3. Since no extra-high power pump source is avaliable, two pump lasers at $1480 \mathrm{~nm}$ with a total power of $600 \mathrm{~mW}$, combined through a polarization beam combiner (PBC), are used as replacement. The $\mathrm{PBC}$ has an insertion loss of $0.7 \mathrm{~dB}$. In order to provide a polarization-diversified pump, the PBC can combine two pump diodes with the same wavelength in an orthogonal plarization state. The C-band EDFA uses $3.6 \mathrm{~m}$ of EDF and $15 \mathrm{~km}$ of DCF. The absorption of erbium doped fiber (EDF) is $11.2 \mathrm{~dB} / \mathrm{m}$ at $980 \mathrm{~nm}$ and $17.5 \mathrm{~dB} / \mathrm{m}$ at $1530 \mathrm{~nm}$. The DCF has loss, dispersion parameter and nonlinear coefficient of $0.4 \mathrm{~dB} / \mathrm{km},-110 \mathrm{ps} / \mathrm{nm} / \mathrm{km}$ and $14.5 \times 10^{-10} \mathrm{~W}^{-1}$, respectively, measured at $1550 \mathrm{~nm}$. While the L-band RFA uses $15 \mathrm{~km}$ of DCF for both dispersion compensation and Raman amplification. DCF has smaller core diameter than standard single-mode fibre (SMF) to provide a larger Raman gain. By the same token, the C-band EDFA uses 15-km DCF for dispersion compensated $100 \mathrm{~km}$ SMF. A narrow linewidth tunable laser, set at $-20 \mathrm{dBm}$ as input power, is swepted from 1530 to $1600 \mathrm{~nm}$ with $5 \mathrm{~nm}$ interval for gain and NF measurements.

\section{Experimental results and discussion}

Fig. 4 (a) shows the measured overall gain spectra for the hybrid $\mathrm{C}+\mathrm{L}$ band EDFA/RFA under various pump splitting ratios. The overall gain is defined as the signal power increased from the input $\mathrm{C} / \mathrm{L}$ WDM coupler to the output C/L WDM coupler, including $6.6 \mathrm{~dB}$ of DCF loss for each band. The total pumping power is $600 \mathrm{~mW}$ combined by two 300-mW polarized-mutliplexed pump lasers. If the insertion loss of $\mathrm{PBC}$ is neglectable, the pump power is divided into $20 \mathrm{~mW}$ for the C-band EDFA and 580 $\mathrm{mW}$ for the L-band RFA, that corresponds to a pump power shared ratio of $1: 29$. Note that the overall gain has a smaller value than the effective Raman gain (i.e., on/off gain) as expected. The total insertion loss is $8.5 \mathrm{~dB}$ at $1550 \mathrm{~nm}$ for fiber components including C/L WDM couplers, 1480/1550 nm WDM coupler and DCF. Note that the loss for DCF in the L band is lager than $0.4 \mathrm{~dB} / \mathrm{km}$. So, the effective gain is at least $8.5 \mathrm{~dB}$ larger than the overall gain for RFA. For PDG measurement, the maximum PDG is $\pm 0.1 \mathrm{~dB}$ for the 
EDFA and $\pm 0.3 \mathrm{~dB}$ for the RFA, respectively. The measured $\mathrm{NF}$ data of the hybrid $\mathrm{C}+\mathrm{L}$ band EDFA/RFA at pump ratio of 1:29 are shown in Fig. 4 (b). For the $\mathrm{C}$ band EDFA, the $\mathrm{NF}$ value ranging from 4.4 to $5.3 \mathrm{~dB}$. The higher NF value at $1535 \mathrm{~nm}$ is attributed to the amplified stimulated emission (ASE) noise. For the L band RFA, the $N F_{e q}$, value ranging from -6.5 to $-3.5 \mathrm{~dB}$. The worst NF at 1600 $\mathrm{nm}$ is attributed to low gain region of RFA.

Regarding the effect of double Rayleigh scattering (DRS) noise, which may be an important impact for high gain (maybe $\sim 20 \mathrm{~dB}$ ) discrete RFA. Since the scattered light has the same wavelength as the signal, an optical spectrum analyzer can't be used to separate them for scattered light measurement. Fortunately, the DRS effect is minor since the overall gain is less than $15 \mathrm{~dB}$. Another proposed scheme to reduce the cost the $\mathrm{C}+\mathrm{L}$ band EDFA/RFA is shown in Fig. 5. The DCF is $50 \%$ saved by locating a $15 \mathrm{~km}$ DCF after the second C/L WDM coupler. In such a scheme, the $\mathrm{L}$ band signals travel along the lower path in order to by pass the lossy medium (EDF) and $\mathrm{C}+\mathrm{L}$ band signals pass through the same DCF. The overall gain and NF characteristics of two fiber amplifiers shown in Fig. 3 and Fig. 5 are similiar to each other except that the RFA is forward pumped in Fig. 5. Nevertheless, the signals suffer small PDG effect since the pump laser pair are launch into the DCF in an orthogonal state.

\section{Conclusion}

A novel hybrid $\mathrm{C}+\mathrm{L}$ band EDFA/RFA is designed to share the $1480 \mathrm{~nm}$ pump laser(s). The concepts are based on three-level amplification mechanism for the $\mathrm{C}$ band EDFA and Raman shift amplification mechanism for the $\mathrm{L}$ band RFA, respectively. It has the advantage to simplify the pump source design. The optimum splitting ratio for the pump power is 1:29 for EDFA/RFA. The hybrid $\mathrm{C}+\mathrm{L}$ band EDFA/RFA, with features of wide bandwidth, flattened gain and low NF, may find vast applications in WDM system and lightwaev transmission.

\section{Acknowledgments}

The work is conducted at National Taiwan University of Science and Technologies. S.-K. Liaw, Y.-L. Hsiao and I.-G. Lai are supported in part by NSC under projects No. 94-2219-E-011-007 and 94-2622-E-011-021-CC3, Taiwan, ROC. We thank David Lan for kindness help, Anteya Cooperation and Chung Hua Telecom Laboratory for suporting the equipments.

\section{References}

[1] Y. Sun, A. K. Srivastava, J. Zhou and and J. W. Sulhoff, "Optical fiber amplifiers for WDM optical networks', Bell Labs Tech. J., vol. 4, pp. 187-206, 1999.

[2] M. N. Islam, "Raman amplifier for telecommunications", IEEE J. Sel. Area Quantum Electron., vol. 8, pp. 848-559, 2002.

[3] M. N. Islam, S. Hwang, K. W. Song, K. U. Song, S. H. Park, J. Nilsson, and K. Cho, "Comparative high power conversion efficiency of C- plus L-band EDFA", Electron. Lett., vol. 37, pp. 1539-1541, 2001.

[4] X. Zhou, C. Lu, P. Shum and T.H. Cheng, "A simplified model and optimal design of a multiwave length backward-pumped Raman amplifier", IEEE Photon. Technol. Lett. vol. 13, pp. $945-947,2001$.

[5] S. Namiki, "Recent Advance in Raman Amplifiers", ECOC'01 Tutorial, October, Amsterdam, pp. 162-181, 2001.

[6] H. Masuda and S. Kawai, "Wide-band and gain-flattened hybrid fiber amplifier consisting of an EDFA and a multi-wavelength pumped Raman amplifier", IEEE Photon. Technol. Lett., vol. 11, pp. 647-649, 1999.

[7] Y. Zhu, C. R. S. Fludger, W. S. LEE, P. Lobb, T. Schilhable and A. Hadjifotiou, "Experimental comparison of all-Raman and Raman/EDFA hybrid amplifications using 40 Gbit/s-based transmissions over $400 \mathrm{~km}$ TW-RS fibre", Electron. Lett., vol. 38, pp. 893-895, 2002.

[8] S.-K. Liaw, Z. M. Hung, F.-C. Hung, Y.-F. Chiang and C.-F Chen "Investigation of dual-pump power-equalized Raman amplifier with 21 dB gain", APOC2002, paper 4905-90, Shanghai, China.

[9] K. Rottwitt, J. Bromage, M. Du and A. J Stentz, "Design of distributed Raman amplifiers", ECOC'00, paper 4.4.1. 2000.

[10] G. P. Agrawal, "Nonlinear Fiber Optics," second edition, academic press, New York, 1995

[11] P. C. Becker, N.A. Olsson and J.R.Simpson, "Erbium-doped fiber amplifiers fundamentals and technology, pp.47, Academic Press. 1999.

[12] G. P. Agrawal, "Nonlinear Fiber Optics," second edition, academic press, New York, 1995

[13] Bristiel, B, Gallion, P. Jaouen, Y. and Pincemin, E., "Intrinsic noise figure derivation for fiber Raman amplifiers from equivalent noise figure measurement," Lightwave Technologies in Instrumentation and Measurement Conference, 135-140, 2004. 


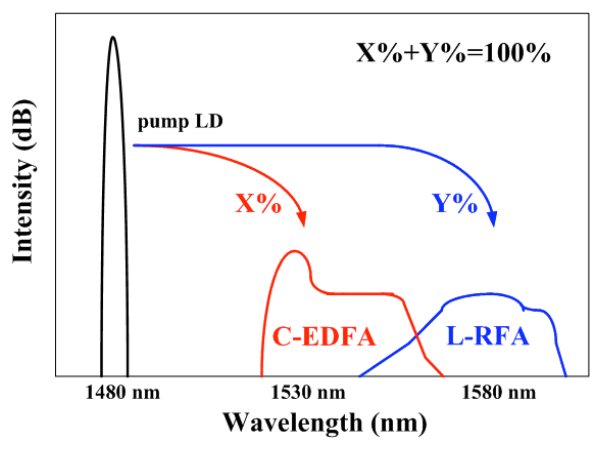

Fig. 1: The concept of using a $1480 \mathrm{~nm}$ pump laser to amplify $\mathrm{C}$ band EDFA and L band RFA amplification simultaneous.

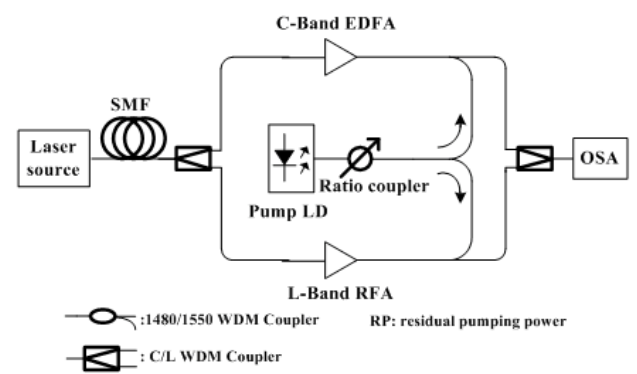

Fig. 2: Schematic diagram of the proposed hybrid $\mathrm{C}+\mathrm{L}$ band EDFA/RFA to share the same pump.

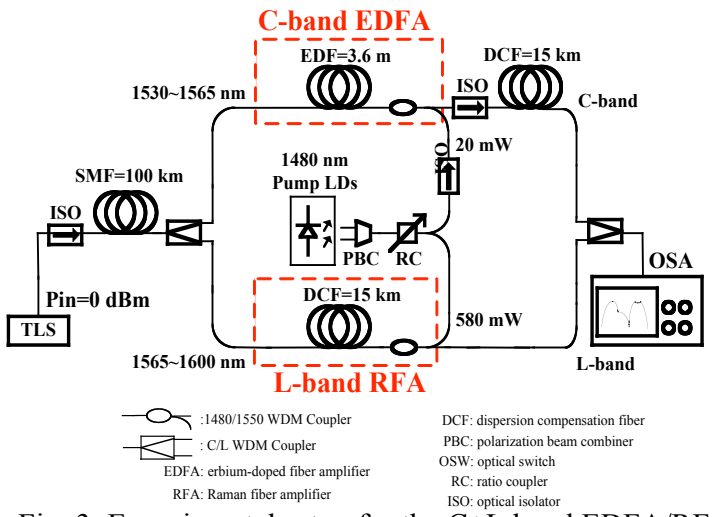

Fig. 3: Experimental setup for the $\mathrm{C}+\mathrm{L}$ band EDFA/RFA.

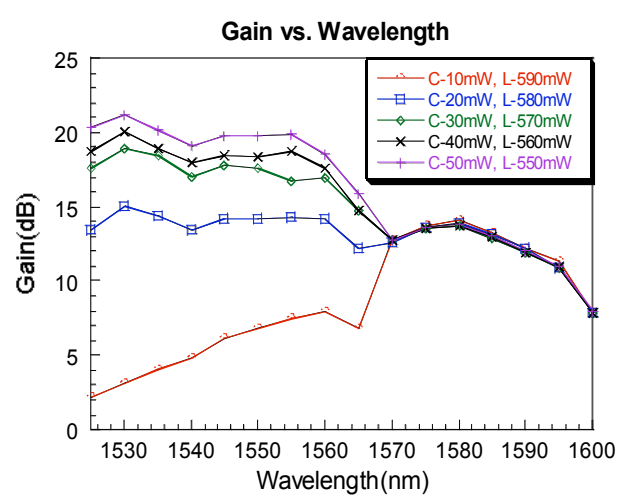

Fig. 4(a)

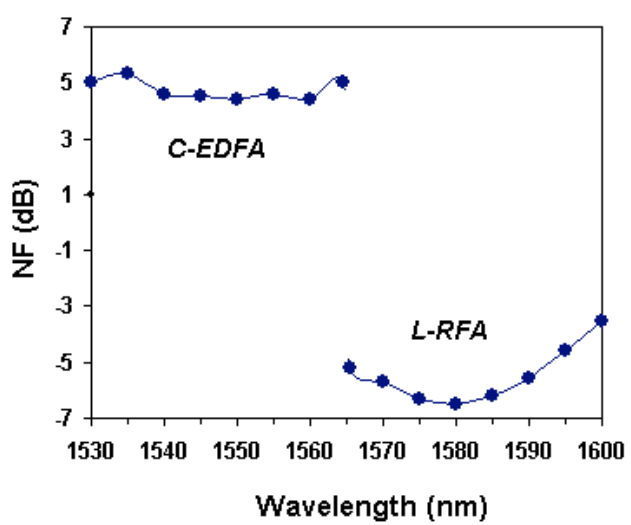

Fig. 4(b)

Fig. 4(a) The measured overall gain spectra for the $\mathrm{C}+\mathrm{L}$ band EDFA/RFA under various pump splitting ratios. (b) Measured $\mathrm{C}+\mathrm{L}$ band noise figure versus wavelength at pump ratio of 1:29.

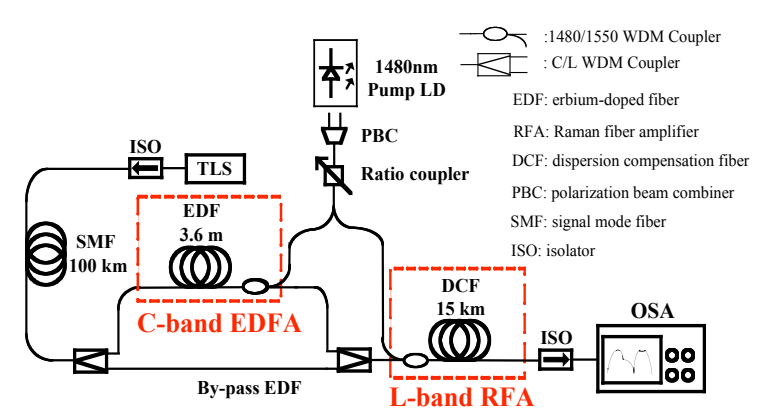

Fig. 5: A proposed scheme to reduce the cost the $\mathrm{C}+\mathrm{L}$ band EDFA/RFA. The DCF is $50 \%$ saved by locating a 15 $\mathrm{km} \mathrm{DCF}$ after the second C/L WDM coupler. 